\title{
Vildagliptin, a DPP4 inhibitor, alleviates diabetes-associated cognitive deficits by decreasing the levels of apoptosis-related proteins in the rat hippocampus
}

\author{
DAN-DAN ZHANG ${ }^{1}$, NAN SHI $^{2}$, HUI FANG $^{1}$, LIANG MA $^{3}$, WEI-PING WU ${ }^{1}$, YA-ZHONG ZHANG $^{1}$, \\ JIN-LI TIAN $^{1}$, LUO-BING TIAN $^{1}$, KANG KANG $^{4}$ and SI CHEN $^{5}$ \\ ${ }^{1}$ Second Department of Endocrinology; ${ }^{2}$ Department of Neurology, Tangshan Gongren Hospital; \\ ${ }^{3}$ Department of Endocrinology, Tangshan Union Medical College Hospital; ${ }^{4}$ Department of Dermatology; \\ ${ }^{5}$ Department of Neurosurgery, Tangshan Gongren Hospital, Tangshan, Hebei 063000, P.R. China
}

Received November 7, 2017; Accepted February 22, 2018

DOI: $10.3892 /$ etm.2018.6016

\begin{abstract}
Cognitive impairment is a prevalent but underestimated complication of diabetes, which can cause spatial memory and learning deficits. In the present study, a streptozotocin-induced type 2 diabetic rat model was employed to investigate the effects of vildagliptin, a new oral hypoglycemic agent that acts by inhibiting dipeptidyl peptidase-4, on diabetes-associated cognitive impairments, as well as the molecular mechanisms involved. The present findings demonstrated that vildagliptin treatment prevented memory impairment and decreased the apoptosis of hippocampal neurons. It also attenuated the abnormal expression of caspase-3, B cell lymphoma-2 (Bcl-2) and Bcl-2 associated X protein in the diabetic model. Vildagliptin treatment also reversed diabetes-induced decreases in phosphorylated (p)-protein kinase B (Akt) and p-glycogen synthase kinase $3 \beta$ (GSK3 $\beta$ ), brain-derived neurotrophic factor and nerve growth factor expression levels. The results indicated that the administration of vildagliptin exerts a protective effect against cognitive deficits by decreasing the expression of apoptosis-related proteins in the hippocampus and that this protective effect was mediated via the Akt/GSK3 $\beta$ signaling pathway.
\end{abstract}

\section{Introduction}

Diabetes mellitus (DM) is a chronic metabolic disorder that can affect multiple organs through its duration. Emerging evidence suggests that DM is linked to an increased risk of mild cognitive

Correspondence to: Professor Hui Fang, Second Department of Endocrinology, Tangshan Gongren Hospital, 27 Wenhua Road, Tangshan, Hebei 063000, P.R. China

E-mail: fanghui2818@126.com

Key words: dipeptidyl peptidase-4 inhibitor, vildagliptin, apoptosis, cognitive deficits, protein kinase $\mathrm{B} / \mathrm{glycogen}$ synthase kinase $3 \beta$ pathway, diabetes mellitus impairment (MCI) and the development of dementia (1). In 1922, Miles and Root noted that diabetes has a detrimental effect on the central nervous system (CNS), which may lead to cognitive dysfunction (2). In 2006, Mijnhout et al proposed the concept of 'diabetes-associated cognitive decline' (DACD) in order to facilitate further research into this disorder (3).

Our previous studies have indicated that patients with type 2 diabetes develop cognitive dysfunction and perform poorly with respect to short term memory, visuospatial/constructional memory, delayed memory, attention and language skills (4). Accumulating evidence suggests that DACD is associated with metabolic dysfunction in the brain (5), deficiencies in nerve growth factor (NGF) and oxidative stress factors, and the inhibition of cell survival signaling pathways (6). However, the underlying mechanisms and potential prevention methods have yet to be elucidated.

The dipeptidyl peptidase-4 (DPP-4) inhibitor vildagliptin is a novel anti-hyperglycemic drug that may benefit patients with diabetes in various ways, including by regulating blood glucose levels with fewer associated adverse events, increased weight loss and cardiovascular advantages (7). Whether it has neuroprotective effects and the potential mechanisms underlying these effects, remains to be studied. The purpose of the present study was to explore whether vildagliptin was able to prevent the development of cognitive deficits in a rodent model of diabetes. Using a diabetic rat model, behavioral tests were performed and the expression levels of protein kinase B (Akt), phosphorylated (p)-Akt, glycogen synthase kinase $3 \beta$ (GSK3 $\beta$ ) and $\mathrm{p}-\mathrm{GSK} 3 \beta$ were measured in the brain, in addition to those of caspase-3, B cell lymphoma-2 (Bcl-2) and Bcl-2 associated $\mathrm{X}$ protein (Bax).

\section{Materials and methods}

Animals. The animal experiments were conducted with the approval of the Ethics Committee of Hebei Medical University (Shijiazhuang, China). A total of 30 male Wistar rats (age, 15 weeks; weight, 220-250 g) were obtained from the Animal Experiment Center of North China University of Science and Technology (Tangshan, China) in the present study. Rats were 
given ad libitum access to water and were maintained at a temperature of $20-24^{\circ} \mathrm{C}$ and a humidity of $50 \pm 10 \%$ with a standard 12-h light/dark cycle. Following 2 weeks of acclimation, 20 Wistar rats were randomly divided into two groups $(n=10)$ : The DM group and the vildagliptin-treated diabetic group. A total of $40 \mathrm{mg} / \mathrm{kg}$ streptozotocin (STZ; Sigma-Aldrich; Merck KGaA, Darmstadt, Germany) was administered via intraperitoneal injection in all rats to induce diabetes. Establishment of the diabetic models were regarded as successful if blood glucose levels were $>16.7 \mathrm{mmol} / \mathrm{l}$. At 10 weeks following the successful establishment of the models, rats in the DM group received a dose-matched placebo (saline) and rats in the vildagliptin-treated group were administered $5 \mathrm{mg} / \mathrm{kg}$ vildagliptin (Galvus; Novartis International AG, Basel, Switzerland) once a day via oral gavage for 4 consecutive weeks. The remaining 10 Wistar rats were treated with an equal amount of normal saline and were designated as the control group. Following treatment, behavioral tests and biochemical experiments were conducted in sequence.

Behavioral tests. Spatial learning and memory were performed using the Morris water maze at 4 weeks following completion of vildagliptin treatments. At the beginning of the navigation task, the rats were placed in a black circular water tank (150 cm in diameter and $60 \mathrm{~cm}$ in depth) and allowed to swim for $5 \mathrm{~min}$. They were trained to find a hidden platform $(14 \mathrm{~cm}$ in diameter), which was located at the midpoint of the target quadrant, at a fixed time each day. During this period, the rats were placed randomly in the water at four different starting points (corresponding to the different quadrants). If the rats successfully found the platform during the Morris water maze test, they would be left on the platform for $15 \mathrm{sec}$ prior to the start of the next training section. On day 5 of the spatial tests, the platform was removed and the rats were placed into the water at the same randomly selected starting points. Maze performance was recorded using a video camera located above the pool and interfaced with a video tracking system (HVS Imaging, Hampton, UK). The mean escape latency of a total of 5 trials and the times for which the rats remained in the quadrant, were then calculated.

Histology. Following completion of the behavioral tests, the hippocampus was excised and frozen. Sections of the hippocampal tissue were investigated for neuronal damage using Nissl's staining method. Rats were anesthetized with sodium pentobarbital (60 mg/kg; Sinopharm Chemical Reagent Co., Ltd., Shanghai, China) and sacrificed via transcardiac perfusion with cold PBS, and subsequently fixed with cold $4 \%$ paraformaldehyde, containing $0.2 \%$ saturated picric acid, in PBS for $24 \mathrm{~h}$ at $4^{\circ} \mathrm{C}$. The brains were removed and the CA1 region of the hippocampus were post-fixed overnight at $4^{\circ} \mathrm{C}$ in the aforementioned fixative solution. The remaining samples were frozen at $-80^{\circ} \mathrm{C}$. Paraffin-embedded tissue sections were cut in the coronal plane at a thickness of $5 \mu \mathrm{m}$ using a microtome. These sections were de-paraffinized with xylene and rehydrated using a descending series of alcohol, stained with $0.1 \%$ (w/v) cresyl violet for $10 \mathrm{~min}$ at $37^{\circ} \mathrm{C}$, and the severity of neuronal damage was evaluated according to the number of surviving neurons. Three Nissl-stained sections from each rat was randomly selected and the number of surviving neurons was counted in three randomly chosen fields. Samples were observed using an optical microscope (magnification, x200; Olympus Corporation, Tokyo, Japan). The mean number of morphologically intact neurons per $100 \mu \mathrm{m}$ was calculated using Image J 1.41 software (National Institutes of Health, Bethesda, MD, USA) in the CA1 hippocampal area in order to estimate the extent of neuronal damage.

Western blotting. Following behavioral tests, the hippocampus of each rat was excised and flash-frozen in liquid nitrogen. Frozen samples in liquid nitrogen were obtained and lysed in Tissue Protein Lysis Solution (Thermo Fisher Scientific, Inc., Waltham, MA, USA) which contained 5\% Proteinase Inhibitor Cocktail (Sigma-Aldrich; Merck KGaA). Protein concentration was determined by using a BCA reagent (OriGene Technologies, Inc., Beijing, China) method. A total of $25 \mu \mathrm{g}$ of extracted protein were resolved via SDS-PAGE and transferred to a polyvinylidene difluoride membrane. The membranes were blocked in 5\% non-fat milk for $2 \mathrm{~h}$ at room temperature and washed three times in PBS with Tween-20. The membranes were probed overnight at $4^{\circ} \mathrm{C}$ with primary antibodies specific for Bcl-2 (1:500; cat. no. BS70205; Biogot Technology Co., Ltd., Nanjing, China) and Bax (1:500; cat. no. BS6420; Biogot Technology Co., Ltd.), and caspase-3 (1:1,000; cat. no. AB13847; Abcam, Cambridge, UK), p-Akt (1:1,000; cat. no. AB38449; Abcam), Akt (1:1,000; cat. no. AB8805; Abcam), GSK3 $\beta$ (1:1,000; cat. no. AB32391; Abcam) and p-GSK3 $\beta$ (1:1,000; cat. no. AB75745; Abcam), brain-derived neurotrophic factor (BDNF; 1:1,000, cat. no. AB226843; Abcam), NGF (1:1,000, cat. no. AB5199; Abcam) and $\beta$-actin (1:1,000; cat. no. AB8227; Abcam), followed by labeling with horseradish peroxidase-conjugated secondary antibodies (1:1,000; cat. no. AB205718; Abcam) at $4^{\circ} \mathrm{C}$ for $2 \mathrm{~h}$. Bands were visualized using an enhanced chemiluminescent (Bio-Rad Laboratories, Inc., Hercules, CA, USA) reagent and were analyzed using ImageJ 1.41 software (National Institutes of Health, Bethesda, MD, USA).

Statistical analysis. All data are presented as the mean \pm standard deviation. Each experiment was repeated a minimum of 3 times. Differences among three or more groups were analyzed using one-way analysis of variance, followed by the Bonferroni post hoc test for multiple comparisons. $\mathrm{P}<0.05$ was considered to indicate a statistically significant difference.

\section{Results}

Vildagliptin ameliorates the impairments to spatial learning and memory observed in the DM group. It was evaluated whether vildagliptin treatment was able to improve spatial learning function using the Morris water maze. As presented in Fig. 1A, STZ induced a significant spatial learning deficit in the DM group as compared with the control group, whereas the administration of vildagliptin significantly reduced the escape latency when compared with the DM group $(\mathrm{P}<0.05)$. Following 4 days of training, the platform was removed. Under these conditions, the time spent in target quadrant was significantly lower for the DM group than for the control group, but was significantly increased in the vildagliptin treatment group compared with the DM group (Fig. 1B). 

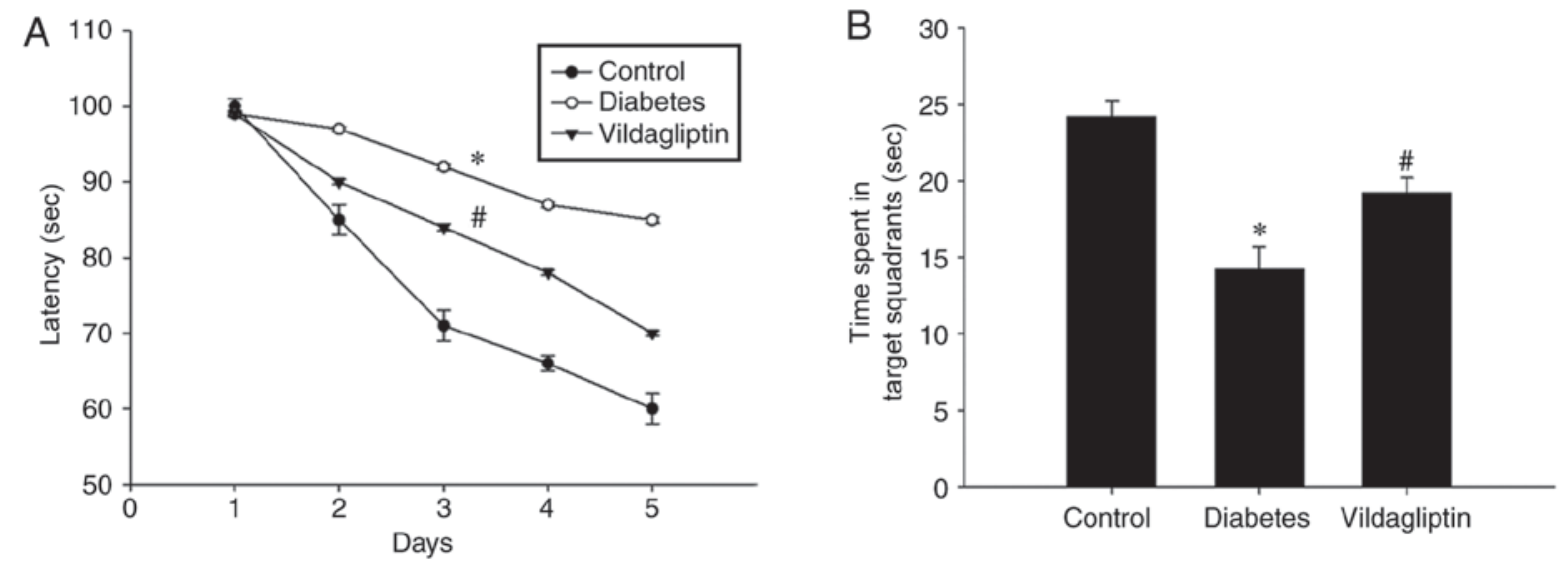

Figure 1. Vildagliptin treatment improved learning and memory in the diabetic rat model. (A) Latency of finding the platform in the Morris water maze during the 4-day training period. (B) Time spent in the target quadrant following removal of the platform. ${ }^{~} \mathrm{P}<0.05$ vs. control; ${ }^{\text {}} \mathrm{P}<0.05$ vs. diabetes. Data are presented as the mean \pm standard deviation $(\mathrm{n}=3)$.
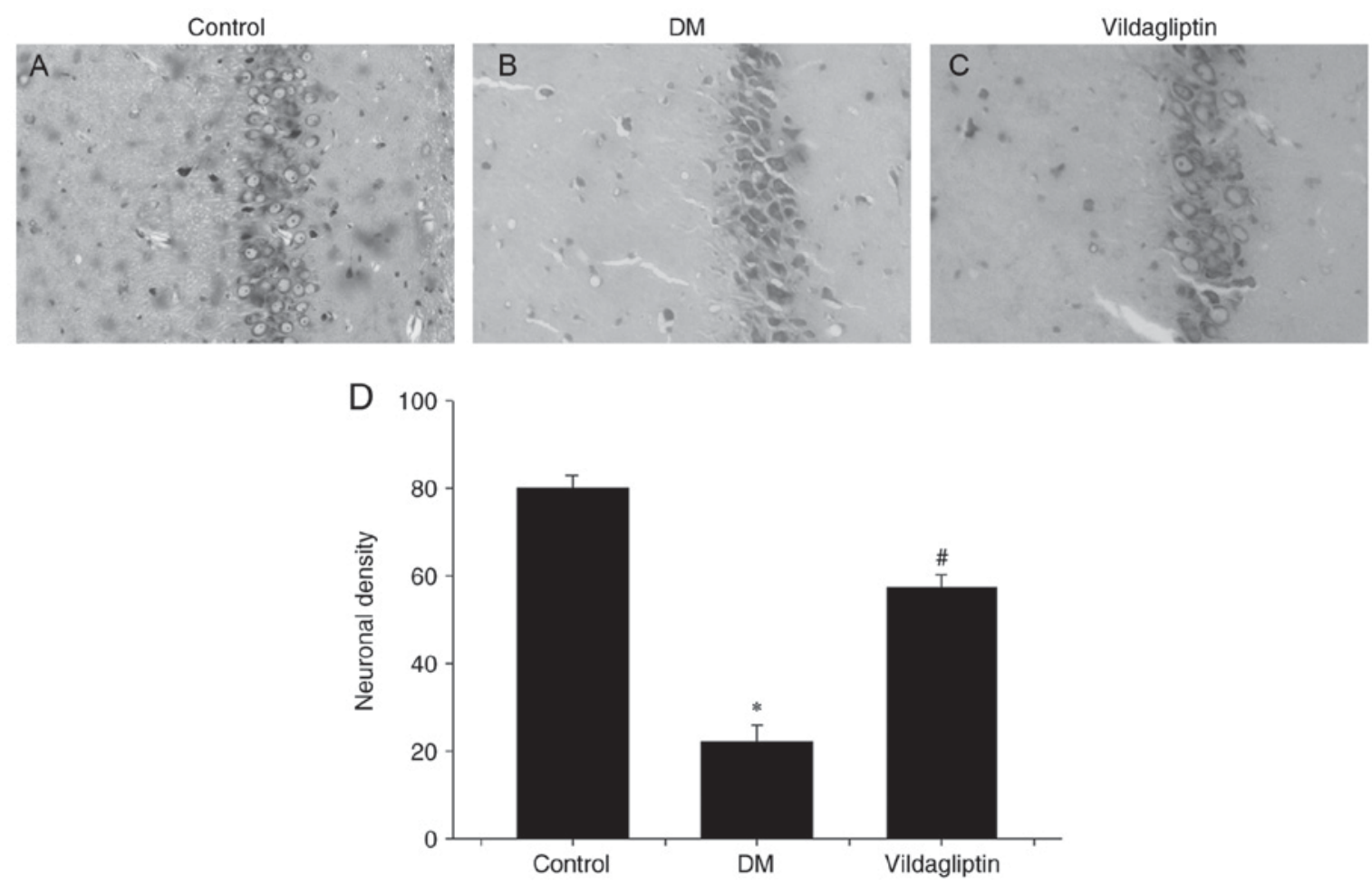

Figure 2. Histological analysis of the effects of vildagliptin on neuronal injury induced by DM in rats. Nissl's staining was performed on tissue sections from the hippocampal CA1 regions (magnification, x20). (A) Neurons with round vesicular nuclei and prominent nucleoli were observed in the control group, with no signs of degeneration. (B) Altered neurons characterized by pronounced shrinkage of the neuronal bodies, loss of nuclei and pyknotic pyramidal cells in the DM group. (C) Neuronal alterations were eliminated in the vildagliptin treatment group. (D) Neuronal density of each group. ${ }^{*} \mathrm{P}<0.05$ vs. control; ${ }^{\prime \prime} \mathrm{P}<0.05$ vs. DM. DM, diabetes mellitus. Data are presented as the mean \pm standard deviation $(n=3)$.

Vildagliptin prevents neuronal cell loss and attenuates abnormalities in caspase-3, Bax and Bcl-2 expression in the diabetic model. Nissl's staining method was performed to investigate neuronal alterations in the hippocampal CA1 region of rats in different groups (Fig. 2). In the DM group, hippocampal neurons were characterized by pronounced shrinkage of the neuronal bodies, with the loss of nuclei and pyknotic pyramidal cells (Fig. 2B). However, neurons in the control group were large, conical-shaped cells with well-demarcated amphophilic cytoplasm and round vesicular nuclei with prominent nucleoli (Fig. 2A). Treatment with vildagliptin reduced the DM-induced cell loss and pyknotic cells, but degenerating cells with altered morphology were still present (Fig. 2C). Vildagliptin exhibited a significant protective ability against DM-induced neurotoxicity (Fig. 2D).

Western blotting was used to detect caspase-3, Bax and Bcl-2 protein levels in the rat hippocampi. Compared with the normal control group, hippocampal caspase-3 was significantly increased in the DM group and significantly downregulated by vildagliptin treatment compared with the DM group (Fig. 3A). As presented in Fig. 3B, the Bax/Bcl-2 ratio in the 
A
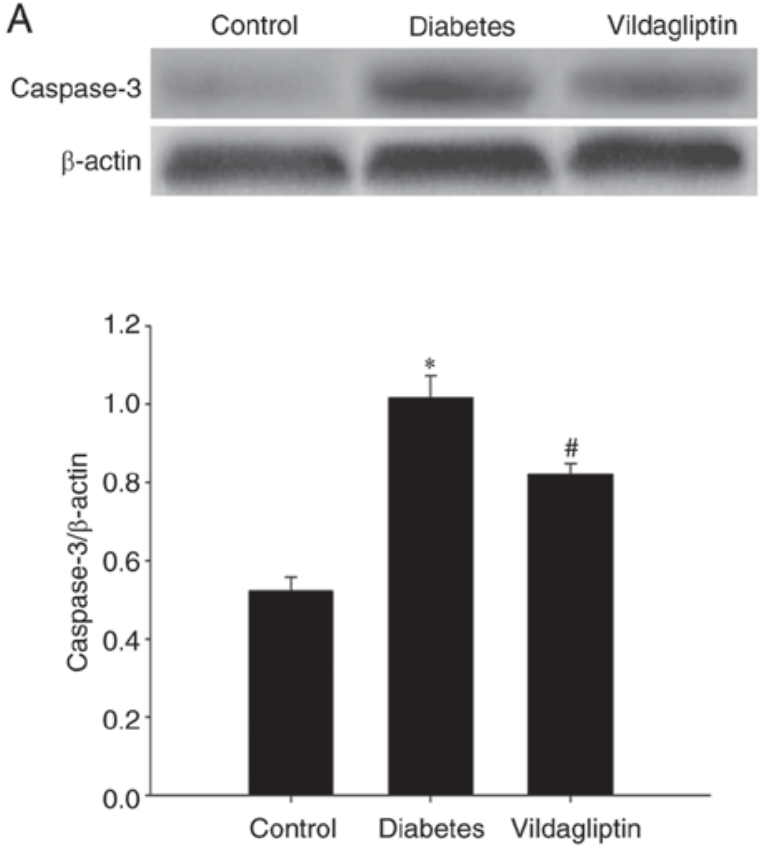

B
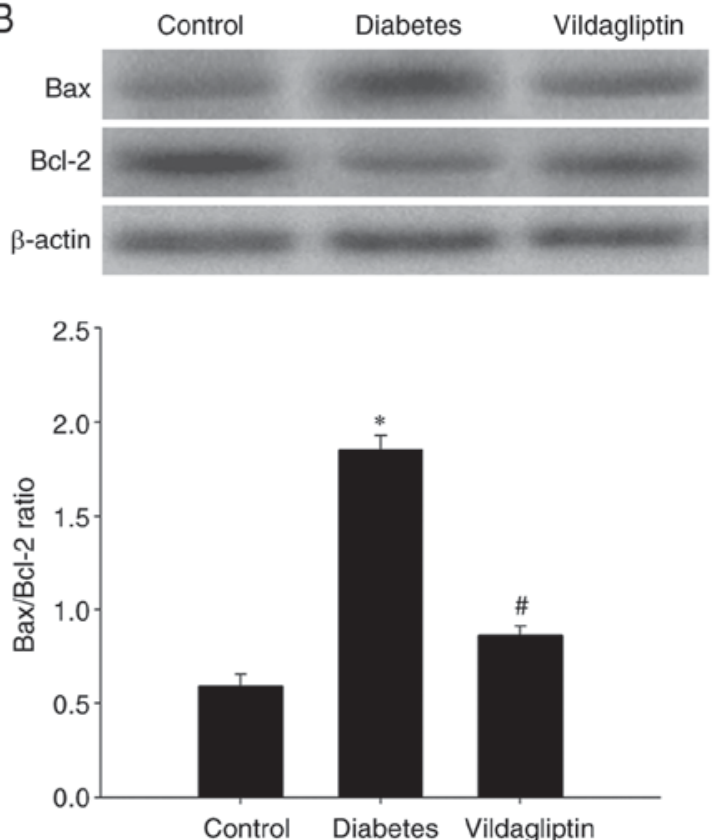

Figure 3. Vildagliptin protects against streptozotocin-induced neuronal apoptosis in the diabetes rat model. Hippocampal tissues from the rats were isolated immediately for western blot analysis, in order to detect the (A) caspase-3, (B) Bax and Bcl-2 expression levels. Data are expressed as the mean \pm standard deviation. "P<0.05 vs. control; " $\mathrm{P}<0.05$ vs. DM. DM, diabetes mellitus. Bcl-2, B cell lymphoma-2; Bax, Bcl-2 associated X protein.

A
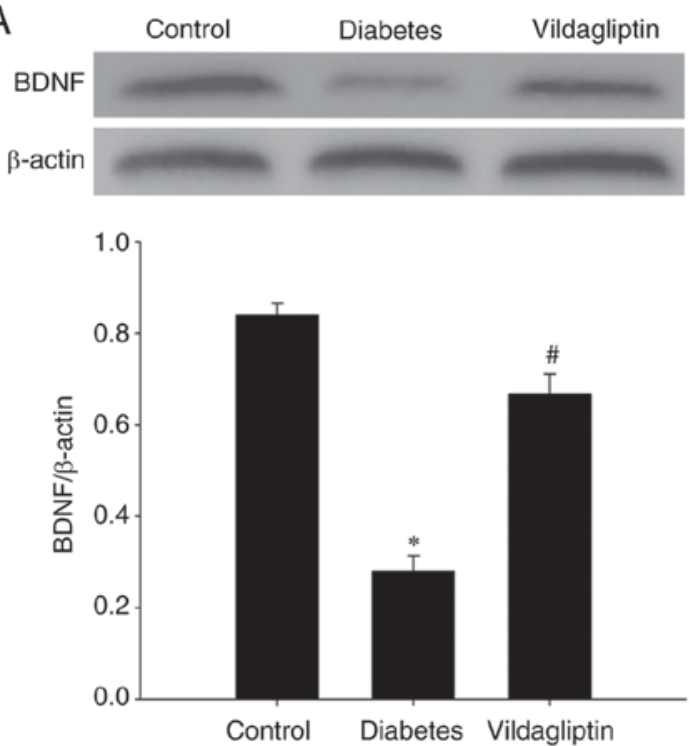

B
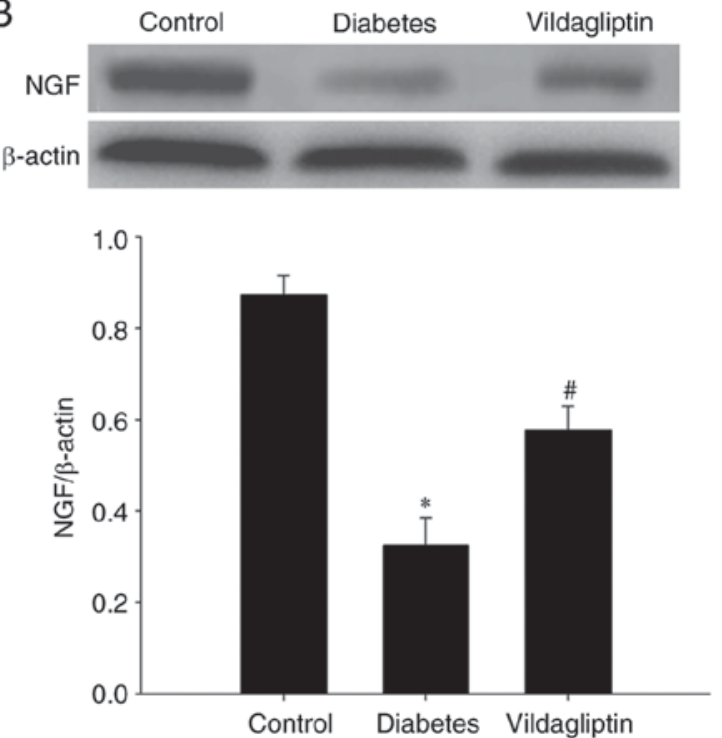

Figure 4. Vildagliptin attenuated the diabetes-induced decreased expression of neurotrophic factors. (A) BDNF and (B) NGF expression levels. Data are expressed as mean \pm standard deviation. " $\mathrm{P}<0.05$ vs. control; ${ }^{\#} \mathrm{P}<0.05$ vs. DM. DM, diabetes mellitus. BDNF, brain-derived neurotrophic factor; NGF, nerve growth factor.

rat hippocampi differed considerably among the three groups. Compared with the control group (0.591 \pm 0.020$)$, STZ-induced $\mathrm{DM}$ significantly increased this ratio $(1.856 \pm 0.021 ; \mathrm{P}<0.05)$, whereas the STZ-induced increases in the $\mathrm{Bax} / \mathrm{Bcl}-2$ ratios were attenuated by treatment with vildagliptin $(0.861 \pm 0.012$; $\mathrm{P}<0.05)$.

Vildagliptin reverses the decrease in neurotrophic factor expression. BDNF and NGF, both neurotrophic factors, were also detected in each group via western blotting. STZ-induced DM significantly reduced the levels of both BDNF and NGF.
By contrast, vildagliptin treatment significantly attenuated the STZ-induced decreases (Fig. 4).

Vildagliptin attenuates the deficit in the Akt/GSK3 $\beta$ pathway. The Akt/GSK3 $\beta$ pathway, which has been proposed to serve an important role in cell apoptosis (8), was investigated in the rats. As presented in Fig. 5, the DM group exhibited significantly decreased $\mathrm{p}$-Akt expression, in addition to significantly increased p-GSK $3 \beta$ expression. Vildagliptin treatment significantly attenuated the decrease in p-Akt expression and decreased the $\mathrm{p}-\mathrm{GSK} 3 \beta$ expression $(\mathrm{P}<0.05)$. By contrast, 
A
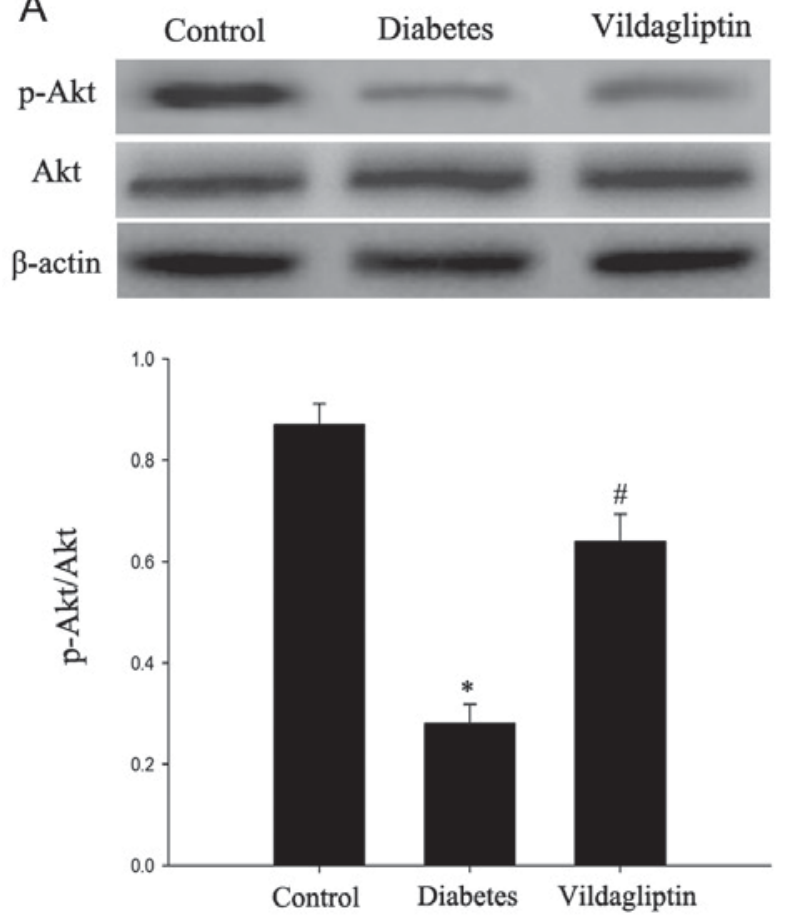

B
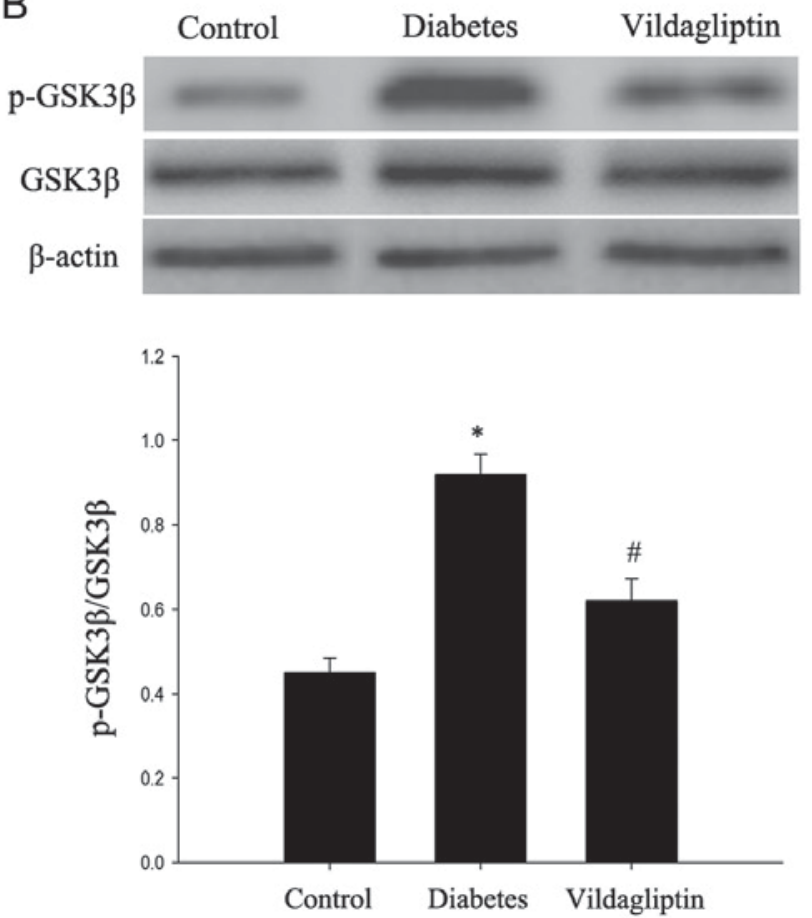

Figure 5. Administration of vildagliptin protects hippocampal neurons from apoptosis via the p-Akt/GSK3ß pathway. (A) Western blotting revealed the relative expression of Akt and p-Akt. (B) Western blotting revealed the relative expression of GSK3 $\beta$ and p-GSK3 $\beta$. Data are expressed as the mean \pm standard

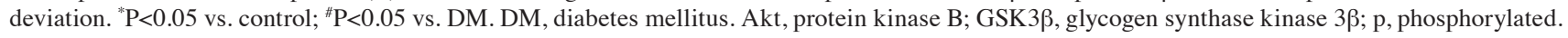

GSK3 $\beta$ and Akt total protein levels did not markedly differ among the three groups.

\section{Discussion}

Cognitive impairment is regarded as one of the most easily underestimated complications of diabetes (9). In the present study, vildagliptin attenuated STZ-induced spatial learning and memory deficits in rats, as evaluated using the Morris water maze. Consistent with the present results, certain prior studies demonstrated that DPP4 inhibitors, including vildagliptin and saxaglipyin may exert notable neuroprotective effects in animal models of neurodegeneration, including stroke (10) and Parkinson's disease (11). Furthermore, DPP4 inhibitors were observed to significantly reduce the plaque load following long-term treatment in a mouse model of Alzheimer's disease (12). In the present study, the pharmacological efficacy of vildagliptin in ameliorating memory impairment was demonstrated in diabetic rats.

DPP4 inhibitors, a new class of anti-diabetic agents, mimic many of the actions ascribed to glucagon-like peptide (GLP)-1 receptor (R) agonists by suppressing DPP4, which act to increase the level of active GLP-1 in the peripheral blood and then indirectly diffuse into the brain (13). Mechanisms of action include the stimulation of insulin and the inhibition of glucagon secretion, and the preservation of $\beta$ cell mass via the stimulation of cell proliferation and the inhibition of apoptosis (14). Unlike GLP-1R agonists, DPP4 inhibitors cannot pass through the blood-brain barrier (15). To date, DPP4 inhibitors for the treatment of diabetes have included sitagliptin, vildagliptin, saxagliptin, alogliptin and linagliptin, among others (16). Vildagliptin is currently available as an efficient treatment agent for type 2 diabetes (17). It has also exhibited neuroprotective properties in several animal models of neurodegenerative disorders (18).

In obese insulin-resistant rats, vildagliptin displayed marked neuroprotective effects $(19,20)$. Vildagliptin administration to STZ-treated rats for a period of 30 days has been demonstrated to result in a significant and dose-dependent reduction of $\mathrm{A} \beta 42$, in addition to alleviating neurotoxicity (21). In rats with STZ-induced Alzheimer's disease, vildagliptin treatment not only reduced amyloid $\beta 42$, but also reduced p-tau, which increases abnormally and accumulates during disease progression. Simultaneously, elevated levels of oxidative stress factors, including tumor necrosis factor- $\alpha$ and interleukin-1 $\beta$, were efficiently reduced following vildagliptin treatment (21). Another previous study tested the neuroprotective effect of vildagliptin on rats with insulin resistance induced by a 12-week high-fat diet (HFD) consumption. The drug effectively attenuated the impairment of brain insulin receptor signaling and improved learning and memory deficits induced by HFD consumption (22).

Further research demonstrated that vildagliptin is able to restore the phosphorylation of neuronal insulin receptor, insulin receptor substance 1 and $\mathrm{Akt} / \mathrm{PKB}$, thus preventing neuronal insulin resistance (23). Furthermore, vildagliptin is able to decrease brain mitochondrial ROS production, mitochondrial membrane potential depolarization and brain mitochondrial swelling in order to ameliorate brain mitochondrial dysfunction (23). Consistent with the present study, Zheng et al (24) proposed that mild cognitive impairment was independently associated with increased DPP4 activity in elderly patients with type 2 diabetes. This phenomenon may be partly due to the effect of DPP4 on inflammation and oxidative stress, 
which may be regarded as an MCI-related risk biomarker (24). Nath et al (25) also reported the effect of vildagliptin on the inhibition of catalase activity.

In addition to inflammation and oxidative stress, neuronal apoptosis is an important type of programmed cell death, which affects cognitive function. It has been documented that GSK3 $\beta$ is associated with the apoptotic pathway and that GSK3 $\beta$ overactivity leads to the increased occurrence of plaques and neuronal loss in neurodegenerative conditions (26). In the present study, downregulation was observed in the levels of p-Akt and an upregulation of p-GSK3 $\beta$ was observed in the hippocampus, whereas caspase-3 expression and the $\mathrm{Bax} / \mathrm{Bcl}-2$ ratio were both increased following the induction of diabetes in the rats. However, vildagliptin attenuated the changes to $\mathrm{p}-\mathrm{Akt}$, p-GSK $3 \beta$ and caspase-3 levels, and to the Bax/Bcl-2 ratio. The Akt/GSK3 $\beta$ signaling pathway is a cell survival pathway that inhibits caspase- 3 and prohibits apoptosis (27). Akt is a serine/threonine protein kinase. PI3K can enhance the activity of p-Akt mediated by phosphoinositide-dependent kinase 1 , which affects the phosphorylation of the downstream protein GSK3 $\beta$. Activation of the Akt/GSK3 $\beta$ pathway can induce apoptosis via phosphorylation of the anti-apoptotic protein myeloid cell leukemia-1, which belongs to the Bcl-2 family (28). Previous research has suggested that the inhibition of GSK $3 \beta$ promotes cell survival; the overexpression of active GSK3 $\beta$ has been demonstrated to promote neuronal apoptosis (29). However, whether the anti-apoptotic effect of vildagliptin is mediated via the Akt/GSK3 $\beta$ pathway requires further verification.

Neurotrophic factors, such as BDNF and NGF, are important regulators involved in plasticity and neuronal cell death $(30,31)$. In the present study, it was discovered that NGF and BDNF were downregulated in the DM model and that vildagliptin treatment reversed this downregulation. A previous study has also indicated that the vildagliptin-induced amelioration of DACD and the neuroprotective effect observed in the current study may be due to increased BDNF and superoxide dismutase activity (32).

In conclusion, the present study demonstrated that vildagliptin may improve the learning and memory deficits induced by diabetes and indicated that decreased levels of apoptosis-related proteins and increased neurotrophic factors may contribute to these effects. In addition, the activation of Akt and the inhibition of GSK3 $\beta$ were conducive to the observed effects of vildagliptin in improving cognitive deficits. Therefore, the present study provided evidence that may facilitate the development of vildagliptin as a preventive or therapeutic agent for diabetes-induced CNS injury. However, the beneficial effects of vildagliptin and associated mechanisms need to be further determined in vivo.

\section{Acknowledgements}

The authors would like to thank all teachers in the Animal Experiment Center of North China University of Science and Technology (Tangshan, China) for their technical support for the experiments.

\section{Funding}

No funding was received.

\section{Availability of data and materials}

All data generated or analysed during this study are included in this published article.

\section{Authors' contributions}

DDZ, NS and HF conceived and designed the study. LM, WPW and YZZ performed the experiments. JLT, LBT and KK wrote the manuscript. DDZ, SC and HF reviewed and edited the manuscript. All authors read and approved the manuscript.

\section{Ethics approval and consent to participate}

All animal experiments were conducted with the approval of the Ethics Committee of North China University of Science and Technology.

\section{Consent for publication}

Not applicable.

\section{Competing interests}

The authors declare that they have no competing interests.

\section{References}

1. Koekkoek PS, Kappelle LJ, van den Berg E, Rutten GE and Biessels GJ: Cognitive function in patients with diabetes mellitus: Guidance for daily care. Lancet Neurol 14: 329-340, 2015.

2. Miles WR and Root HF: Psychologic tests applied to diabetic patients. Arch Intern Med (Chic) 30: 767-777, 1922.

3. Mijnhout GS, Scheltens P, Diamant M, Biessels GJ, Wessels AM, Simsek S, Snoek FJ and Heine RJ: Diabetic encephalopathy: A concept in need of a definition. Diabetologia 49: 1447-1448, 2006.

4. Zhang XY, Liang J, Chen DC, Xiu MH, Yang FD, Kosten TA and Kosten TR: Low BDNF is associated with cognitive impairment in chronic patients with schizophrenia. Psychopharmacology (Berl) 222: 277-284, 2012.

5. Svichar N, Shishkin V, Kostyuk E and Voitenko N: Changes in mitochondrial $\mathrm{Ca}^{2+}$ homeostasis in primary sensory neurons of diabetic mice. Neuroreport 9: 1121-1125, 1998.

6. MacGibbon GA, Cooper GJ and Dragunow M: Acute application of human amylin, unlike beta-amyloid peptides, kills undifferentiated PC12 cells by apoptosis. Neuroreport 8: 3945-3950, 1997.

7. Ji LN, Pan CY, Lu JM, Li H, Zhu DL, Li Q, Li QF, Peng YD, Tian HM, Yao C, Zhao ZG, et al: Efficacy and safety of combination therapy with vildagliptin and metformin versus metformin uptitration in Chinese patients with type 2 diabetes inadequately controlled with metformin monotherapy: A randomized, open-label, prospective study (VISION). Diabetes Obes Metab 18: 775-782, 2016.

8. Gao C, Liu Y, Jiang Y, Ding J and Li L: Geniposide ameliorates learning memory deficits, reduces tau phosphorylation and decreases apoptosis via GSK3 $\beta$ pathway in streptozotocin-induced alzheimer rat model. Brain Pathol 24: 261-269, 2014

9. Selvarajah D and Tesfaye S: Central nervous system involvement in diabetes mellitus. Curr Diab Rep 6: 431-438, 2006.

10. Darsalia V, Olverling A, Larsson M, Mansouri S, Nathanson D, Nyström T, Klein T, Sjöholm $\AA$ and Patrone C: Linagliptin enhances neural stem cell proliferation after stroke in type 2 diabetic mice. Regul Pept 190-191: 25-31, 2014.

11. Nassar NN, Al-Shorbagy MY, Arab HH and Abdallah DM: Saxagliptin: A novel antiparkinsonian approach. Neuropharmacology 89: 308-317, 2015. 
12. D'Amico M, Di Filippo C, Marfella R, Abbatecola AM Ferraraccio F, Rossi F and Paolisso G: Long-term inhibition of dipeptidyl peptidase-4 in Alzheimer's prone mice. Exp Gerontol 45: 202-207, 2010.

13. Pintana H, Apaijai N, Chattipakorn $\mathrm{N}$ and Chattipakorn SC: DPP-4 inhibitors improve cognition and brain mitochondrial function of insulin-resistant rats. J Endocrinol 218: 1-11, 2013.

14. Deacon CF: Therapeutic strategies based on glucagon-like peptide 1. Diabetes 53: 2181-2189, 2004.

15. Deacon CF: Dipeptidyl peptidase-4 inhibitors in the treatment of type 2 diabetes: A comparative review. Diabetes Obes Metab 13: 7-18, 2011.

16. Messori A, Fadda V, Maratea D, Trippoli S and Marinai C: Testing the therapeutic equivalence of alogliptin, linagliptin, saxagliptin, sitagliptin or vildagliptin as monotherapy or in combination with metformin in patients with type 2 diabetes. Diabetes Ther 5: 341-344, 2014.

17. Drucker DJ and Nauck MA: The incretin system: Glucagon-like peptide-1 receptor agonists and dipeptidyl peptidase-4 inhibitors in type 2 diabetes. Lancet 368: 1696-1705, 2006.

18. Matteucci E and Giampietro O: Mechanisms of neurodegeration in type 2 diabetes and the neuroprotective potential of dipeptidyl peptidase 4 inhibitors. Curr Med Chem 22: 1573-1581, 2015.

19. Pintana H, Tanajak P, Pratchayasakul W, Sa-nguanmoo P, Chunchai T, Satjaritanun P, Leelarphat L, Chattipakorn N and Chattipakorn SC: Energy restriction combined with dipeptidyl peptidase-4 inhibitor exerts neuroprotection in obese male rats. Br J Nutr: 1-9, 2016.

20. Sripetchwandee J, Pipatpiboon N, Pratchayasakul W, Chattipakorn $\mathrm{N}$ and Chattipakorn SC: DPP-4 inhibitor and PPAR $\gamma$ agonist restore the loss of CA1 dendritic spines in obese insulin-resistant rats. Arch Med Res 45: 547-552, 2014.

21. Kosaraju J, Murthy V, Khatwal RB, Dubalab A, Chinnic S, Muthureddy Nataraj SK and Basavan D: Vildagliptin: An anti-diabetes agent ameliorates cognitive deficits and pathology observed in streptozotocin-induced Alzheimer's disease. J Pharm Pharmacol 65: 1773-1784, 2013.

22. Pipatpiboon N, Pratchayasakul W, Chattipakorn N and Chattipakorn SC: PPAR $\gamma$ agonist improves neuronal insulin receptor function in hippocampus and brain mitochondria function in rats with insulin resistance induced by long term high-fat diets. Endocrinology 153: 329-338, 2012.

23. Pipatpiboon N, Pintana H, Pratchayasakul W, Chattipakorn N and Chattipakorn SC: DPP4-inhibitor improves neuronal insulin receptor function, brain mitochondrial function and cognitive function in rats with insulin resistance induced by high-fat diet consumption. Eur J Neurosci 37: 839-849, 2013.
24. Zheng T, Qin L, Chen B, Hu X, Zhang X, Liu Y, Liu H, Qin S, Li G and Li Q: Association of plasma DPP4 activity with mild cognitive impairment in elderly patients with type 2 diabetes: Results from the GDMD study in China. Diabetes Care 39: 1594-1601, 2016

25. Nath S, Ghosh SK and Choudhury Y: A murine model of type 2 diabetes mellitus developed using a combination of high fat diet and multiple low doses of streptozotocin treatment mimics the metabolic characteristics of type 2 diabetes mellitus in humans. J Pharmacol Toxicol Methods 84: 20-30, 2017.

26. Proctor CJ and Gray DA: GSK3 and p53-is there a link in Alzheimer's disease? Mol Neurodegener 5: 7, 2010.

27. Hu S, Begum AN, Jones MR, Oh MS, Beech WK, Beech B, Yang F, Chen P, Ubeda OJ, Kim PC, et al: GSK3 inhibitors show benefits in an Alzheimer's disease (AD) model of neurodegeneration but adverse effects in control animals. Neurobiol Dis 33: 193-206, 2009.

28. Ragot K, Delmas D, Athias A, Nury T, Baarine M and Lizard G: $\alpha$-Tocopherol impairs 7-ketocholesterol-induced caspase-3dependent apoptosis involving GSK-3 activation and $\mathrm{Mcl}-1$ degradation on $158 \mathrm{~N}$ murine oligodendrocytes. Chem Phys Lipids 164: 469-478, 2011.

29. Ambacher KK, Pitzul KB, Karajgikar M, Hamilton A, Ferguson SS and Cregan SP: The JNK- and AKT/GSK3 $\beta$ signaling pathways converge to regulate puma induction and neuronal apoptosis induced by trophic factor deprivation. PloS One 7: e46885, 2012.

30. Qiao HJ, Li ZZ, Wang LM, Sun W, Yu JC and Wang B: Association of lower serum Brain-derived neurotrophic factor levels with larger infarct volumes in acute ischemic stroke. J Neuroimmunol 307: 69-73, 2017.

31. Li R, Ma J, Wu Y, Nangle M, Zou S, Li Y, Yin J, Zhao Y, Xu H, Zhang $\mathrm{H}$, et al: Dual delivery of NGF and bFGF coacervater ameliorates diabetic peripheral neuropathy via inhibiting schwann cells apoptosis. Int J Biol Sci 13: 640-651, 2017.

32. El Batsh MM, El Batch MM, Shafik NM and Younos IH: Favorable effects of vildagliptin on metabolic and cognitive dysfunctions in streptozotocin-induced diabetic rats. Eur J Pharmacol 769: 297-305, 2015. 